\title{
Monogenic Very Early Onset Inflammatory Bowel Disease in Children. A Report of Two Cases and Literature Review
}

\begin{abstract}
Shaden Al Mahamed* and Abdelhai Hammo
Department of Pediatric, Section of Pediatric Gastroenterology, Hepatology, and Nutrition, King Fahad Specialist Hospital, Dammam, Saudi Arabia

*Corresponding Author: Shaden Al Mahamed, Pediatric Gastroenterologist and hepatologist. Department of pediatrics, section of pediatric gastroenterology, hepatology, and nutrition. King Fahad Specialist Hospital, Dammam, Saudi Arabia.
\end{abstract}

Received: May 11, 2020

Published: May 27, 2020

(C) All rights are reserved by Shaden Al

Mahamed and Abdelhai Hammo.

\begin{abstract}
Objective: Patients with a diverse spectrum of rare genetic disorders can present with very early onset inflammatory bowel disease (VEO-IBD), which known as monogenic IBD. We report two patients with very early presentation of Crohn's disease and ulcerative colitis during early childhood and infancy. Rare genetic mutations in the GUCY2C AND AIRE gene respectively were detected in our patients. The early diagnosis of monogenic VEO-IBD allowed for appropriate management.

Method and Patients: Charts of two pediatric patients with confirmed monogenic disorders and VEO-IBD were reviewed retrospectively at King Fahad Specialist Hospital in Dammam, Saudi Arabia.

Result: First Patient was a female patient diagnosed with early onset Crohn's disease under the age of 6 years with underlying congenital familial diarrhea. GUCY2C gene mutation was detected based on whole exome sequencing (WES). Second patient was diagnosed at the age of 8 months with infantile ulcerative colitis associated with ankle joint arthritis and metaphyseal dysplasia. WES detected a mutation in AIRE gene and was consistent with the diagnosis of autoimmune polyendocrinopahty candidiasis ectodermal disorder syndrome type I (APECED).

Conclusion: Monogenic IBD in patients with VEO-IBD is a different disease entity from pediatric IBD. Whole exome sequencing (WES) and targeted genetic panel allow the prompt diagnosis and promote an early appropriate management. Our report of the two rare gene mutations associated with monogenic IBD should contribute to the literature data and increase awareness of monogenic immune defects as a cause of VEO-IBD in children.

Keywords: Very-Early Onset Inflammatory Bowel Disease; VEO-IBD; Crohn's Disease; Ulcerative Colitis; Children; Familial Diarrhea; Diarrhea Type 6; APECED Type1; GUCY2C Gene; AIRE Gene
\end{abstract}

\section{Background and Objective}

Patients with a diverse spectrum of rare genetic disorders can present with very early onset inflammatory bowel disease (VEOIBD), known as monogenic IBD, where a single abnormal gene is responsible for the disease below the age of 6 years [2]. Monogenic IBD is a distinct disease entity and behaves differently from the conventional pediatric IBD in adolescents or adults. It is often presented with severe phenotype form and difficult to treat disease $[1,2]$. Till current, almost 60 genetic disorders have been identified in the literature with associated IBD-like intestinal inflammation. While in conventional IBD (polygenic disorders) above the age of 6 years, hundreds of susceptibility loci encompassing about 300 potential candidate genes which were contributed to the overall disease risk [2]. Whole exome sequencing (WES) and targeted genetic panel allow the prompt diagnosis of monogenic disorder in VEO-IBD patients and result in early appropriate management [3]. The incidence of pediatric IBD has been increasing in Western countries, with annual incidence of 2.2 to 13.3 per 100,000 children [4]. Incidence rate of pediatric IBD in Saudi Arabia between (2003 - 2012) reported as 0.47 per 100,000 individuals, and time trend analysis indicates significant increase in incidence rate over time [5]. Studies from North America, Europe, and Australia reported a prevalence of VEO-IBD that ranges (4\% to 15\%) of pediatric IBD. However, in Saudi Arabia a multicenter national study reported the highest prevalence of VEO-IBD (21.6\%) and infantile onset IBD (9\%) of pediatric IBD [6]. High consanguinity rate in Saudi society might confer genetic susceptibility to early development of IBD.

We reported two Saudi patients with very early onset IBD presented during early childhood and infancy, along with endoscopic and histologic features of Crohn's disease and ulcerative colitis. Rare genetic mutations in the (GUCY2C AND AIRE gene) respectively, were detected in our patients. The early diagnosis of monogenic VEO-IBD allowed for appropriate management. 


\section{Method and Patients}

Charts of 2 pediatric patients with confirmed monogenic disorders and VEO-IBD were reviewed retrospectively, at King Fahad Specialist Hospital in Dammam, eastern province of Saudi Arabia.

\section{Case 1}

First Patient was a 7 years old Saudi female, a product of consanguineous marriage with normal birth weight, had a history of severe chronic secretary diarrhea started at the $1^{\text {st }}$ month of life and resulted in frequent dehydration, high electrolytes loss in the stool (Cl $107 \mathrm{mmol} / \mathrm{l}$, Na $72 \mathrm{mmol} / \mathrm{l}, \mathrm{K} 25 \mathrm{mmol} / \mathrm{l}$ ), and hypokalemia. The condition was associated with failure to thrive. There was no fever, respiratory symptoms, vomiting, or other systemic involvement. She was underweight (weight of $16 \mathrm{~kg}$ ) and had distended abdomen and mouth ulcers by clinical exam with no other clinical abnormalities. Diagnostic laboratory testing for congenital chloride diarrhea, cystic fibrosis, and immunodeficiency disorders were negative.

Endoscopy at the age of 2.5 year revealed normal EGD (upper endoscopy) with normal duodenal biopsy. Colonoscopy revealed chronic nonspecific inflammation, picture of colitis with prominent eosinophilia, and lymphoid hyperplasia in the terminal ileum. Findings were suggestive of inflammatory bowel disease at early age. At the age of 4 years patient started to have frequently bloody diarrhea, and had recurrent abdominal pain, fever, and markedly high inflammatory markers. ASCA was positive, but $\mathrm{p}$ ANCA negative. Follow up colonoscopy at age of 4 years showed severe colitis as in figure 1. Colonic biopsy showed chronic active colitis, cryptitis, but no granulomas, and chronic active ileitis suggestive of Crohn's disease. Ileal mural thickening by MRE (Magnetic Resonance Enterography) was detected subsequently at age of 6 years. Our patient was diagnosed with a full picture of VEO ileo-colonic Crohn's disease. Genetic study of Whole Exome Sequencing (WES) detected a heterozygous variant c.784C $>$ T p.(A rg262) in GUCY2C gene which is reported in the literature as pathogenic mutation found in Familial diarrhea (diarrhea type 6) and associated with increased susceptibility to VEO-IBD. Steroid therapy was required intermittently throughout the course of the disease, in addition to mesalamine. Improvement of the blood in the stool and normalization of inflammatory markers (CRP-ESR) were partially achieved. Lately Anti-TNF- alpha therapy (infliximab) was prescribed and induced colonic mucosal healing and controlled the disease activity However, her secretory diarrhea persisted and oral electrolytes supplement was needed on daily basis.

Case 2

Second patient was a 1 year old Saudi male, a product of consanguineous marriage, presented at age of 8 months with bleeding per rectum, persistent diarrhea, repeated fever, abdominal pain, and high inflammatory markers. Clinical examination was consistent with an underweight child (weight of $6 \mathrm{~kg}$ ) with distended ab-

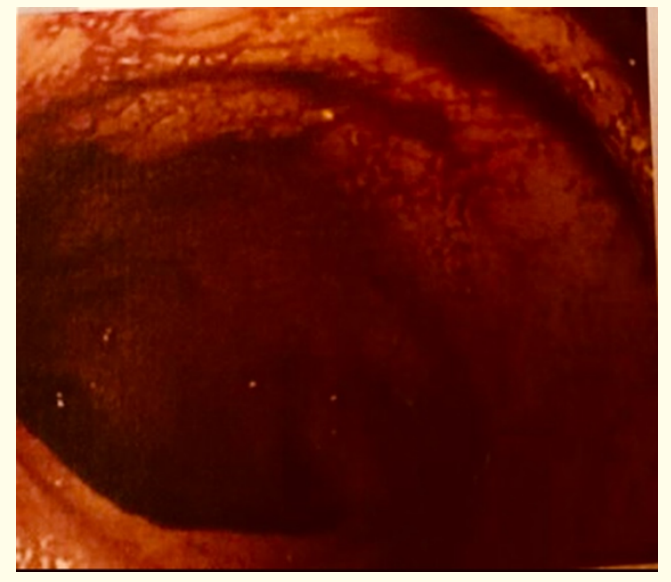

Figure 1: Sever colitis.

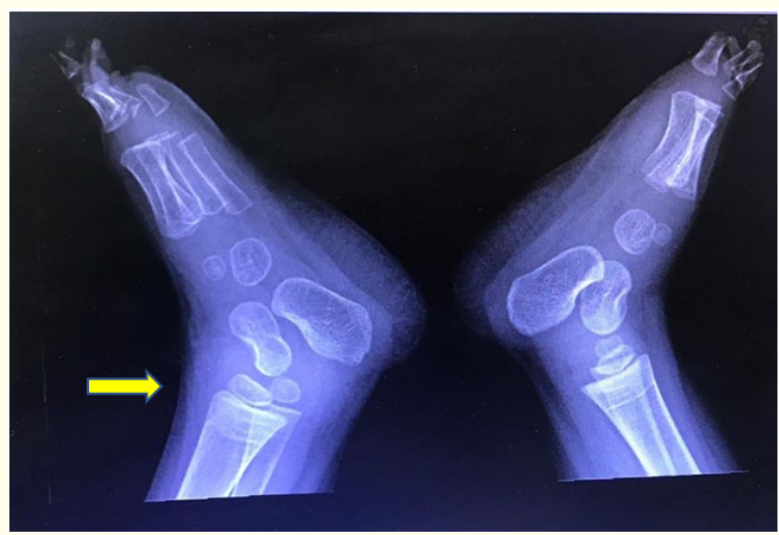

Figure 2: Left ankle arthritis with metaphyseal dysplasia changes.

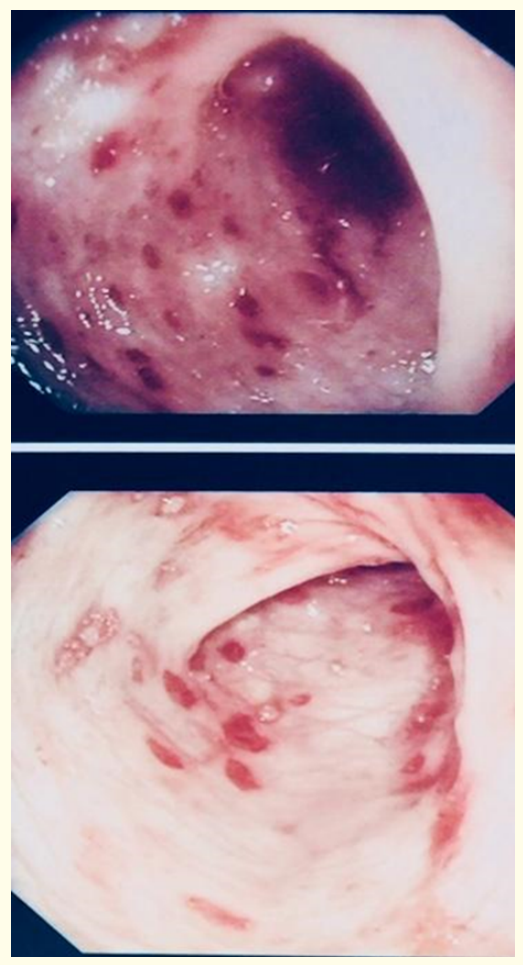

Figure 3: Pancolitis with ulcerations. 
domen, left ankle swelling but no other abnormalities. He had left ankle joint arthritis, joint effusion with synovitis and metaphyseal abnormalities (Figure 2). Infectious work up for colitis and celiac profile were negative, immunodeficiency work up included immunoglobulins level, lymphocytes subset, NBT, IL 10 gene, were normal. Endoscopy was performed at the age of 9 months revealed normal EGD (upper endoscopy) with normal gastric and duodenal biopsy which excluded an autoimmune enteropathy. Colonoscopy showed severe pancolitis with ulcerations, Mayo score of $2 / 3$ (Figure 3). Focal chronic active colitis and cryptitis was found in biopsy result consistent with ulcerative colitis. Further testing of fecal calprotectin in the stool was significantly high $(450 \mu \mathrm{g} / \mathrm{g})$. P ANCA positive, ASCA positive. ANA negative. Capsule endoscopy was not possible due to patient size. However, radiological findings showed no small bowel involvement. Genetic study (WES) detected a heterozygous pathogenic variant mutation c.653-6_653-4del in AIRE gene consistent with the diagnosis of autoimmune polyendocrinopathy candidiasis ectodermal disorder type I (APECED) With reversible or irreversible metaphyseal dysplasia. Parenteral carrier testing confirmed the carrier status on his healthy mother for the same mutation in the AIRE gene, clarifying that the mutation in our patient was inherited and not of a de novo nature. He was started on steroid therapy (oral prednisolone) in addition to mesalamine (5-ASA). The treatment induced clinical and laboratory remission in form of resolution of the bloody diarrhea and fever, normalization of the inflammatory markers (CRP-ESR) and further decrease in the fecal calprotectin level in the stool. However, his symptoms recurred after steroid was tapered and discontinued. Infliximab therapy was on board but still under discussion with the family due to family due to being so young. Ankle joint arthritis required intra articular steroid injection and showed significant improvement.

\section{Discussion}

Children presenting with very early onset IBD may have monogenic basis. Several genetic defects that disturb intestinal epithelial barrier function or affect immune function have been noted in patients from the young age groups and result in intestinal like inflammation $[4,6]$. Familial diarrhea in children is caused by an activating GUCY2C mutation which result in hyperactivation of the CFTR regulator, impaired bacterial sensing, impaired ion homeostasis, and increased loss of sodium and chloride in gut lumen [7]. Patient with familial diarrhea and GUCY2C mutation has increased susceptibility to very early onset IBD. In our first case, a diagnosis of monogenic IBD with underlying congenital familial diarrhea was established based on confirmatory genetic study. Genetic and transcriptional analysis of IBD-associated pathways in patients with GUCY2C-linked familial diarrhea were studied by Rune [8]. The GC- $C$ gene set showed a significant enrichment of association with IBD by Gene Set Enrichment Analysis in IBD genome-wide association data (GWAS). Risk variants in Nucleotide-binding oligomerization domain-containing protein 2 (NOD2) were found in
7/8 FGDS patients with concomitant Crohn's disease. NOD2 plays a key role in the regulation of microbiota in the small intestine [8]. The above findings by Rune's study probably explained well the rationale behind the susceptibility of patients with familial diarrhea to VEO-IBD. Furthermore, A GUCY2C mutation, p.S840I, has been described in 32 affected members of a large Norwegian family with $\mathrm{AD}$ inherited infantile onset diarrhea, frequently associated with IBD. IBD was diagnosed in $20-25 \%$ of these patients [9]. Müller T reported 4 patients with GUCY2C mutations presented with severe congenital secretory diarrhea, one patient with mutation in GC-C developed IBD and arthritis at 4 years of age [7].

The diagnosis of very early onset ulcerative colitis with underlying AIRE gene mutation was established in the second case. Autoimmune regulator protein (AIRE) gene mutation was reported in the literature as one of the immune-dysregulation pathogenesis which might manifest as VEO-IBD in children [10]. AIRE protein Expressed in thymus and peripheral Lymphoid tissue and is responsible for central immunotolerance through negative selection of auto-reactive T-cells. In the APECED thymus, AIRE defect allows for the autoreactive T cells to escape from thymus, and attack selfantigens resulting in autoimmunity [11]. APECED type I is a rare disease in pediatric age group and characterized by a wide variability of the clinical expression and no strong genotype-phenotype correlation has been found among several populations. It has been originally defined by the presence of two of the three most common features: chronic mucocutaneous candidiasis, hypoparathyroidism, and Addison's disease. Classical APECED type 1 is mainly inherited in an autosomal recessive manner with homozygous mutation in AIRE gene. However, a "non-classical APECED" characterized by dominant heterozygous mutations in AIRE gene was reported in the literature by Lucia De martino [11]. Our patient has no endocrine involvement yet which could be explained by the young age of our patient, taking into account the heterogenicity of the disease phenotype and the evolving nature of the APECED type I syndrome.

APECED with AIRE gene mutation and susceptibility to VEO-IBD was described previously in the literature [12]. Patient with AIRE gene mutation showed to have increased in (ASCA) antibodies against Saccharomyces cerevisiae $(\mathrm{p}<0.0001)$ in a pattern reminiscent of Crohn's disease. The high level of (ASCA) antibodies has an inverse correlation with the FOXP3 expression level in T-reg cell. Furthermore, in previous studies the numbers of FOXP3+ cells in gut biopsies were found to be decreased in patient with APECED type 1 in adult population. Since FOXP3 is the master gene driving CD4(+) CD25(+) T cell regulatory (Treg) function, a decrease expression of FOXP3 on $\mathrm{T}$ reg cell in APECED patients results in a failure of T-reg tolerance to the gut flora. Thus, AIRE regulates indirectly gut homeostasis in regard the impact of microbiota in autoimmunity [12]. In our patient, the mesalamine (5-ASA) and steroid therapy improved the symptoms and the bloody diarrhea but no colonic mucosal healing was achieved. Biological therapy (Anti-TNF therapy) was under discussion due to the young age of 
the patient. The question remains as to whether the infliximab can help in achieving a colonic mucosal healing in a case of VEO-IBD with underlying immune-dysregulation status. Moreover, precision therapy to specific genetic defects needs to be taken into account crucially to employ a targeted therapy. In a recent review by Judith, a less robust response to Infliximab (IFX) in children with VEO-IBD compared with older children was seen in a recent study of 42 patients with VEO-IBD compared with 130 children with older onset IBD $(\mathrm{P}<0.01)$. These findings are reflective of the poorer response to IFX in the very young children as compared with older patients with IBD [13].

In the literature few reported cases of APECED type I associated with arthritis or metaphyseal dysplasia as our case [14,15]. The data in the literature for APECE D type I with pediatric IBD is very scarce. Johnson et al in his review had discussed the role of defective T-cell immune tolerance in the autoimmunity and predisposition for VEO-IBD. AIRE gene (AR-AD inheritance) was one of the monogenic defects which enrolled in VEO-IBD pathway [10]. Our case is one of the rare reported cases for the role of mutated AIRE gene in pediatric patient with very early onset IBD, further research and investigation in pediatric population are warranted.

\section{Conclusion}

Monogenic VEO-IBD is a distinct disease entity, often presenting with severe phenotype and difficult to treat disease. We reported two Saudi patients with very early onset IBD during early childhood and infancy. Crohn's disease was diagnosed on very early age in the first patient with underlying familial diarrhea disorder and rare genetic mutations in the GUCY2C gene. Infantile ulcerative colitis was diagnosed in the second patient with underlying APECED type 1 (non-classical) and rare mutation in the AIRE gene. Whole exome sequencing (WES) allows the prompt diagnosis and promotes early and appropriate management for monogenic IBD. Targeted genetic panel might be the gold standard for diagnosis of monogenic disorder whenever is available. Our report should contribute to the literature data and increase the awareness of monogenic immune defects as a cause of VEO-IBD in children.

\section{Conflict of Interest}

There is no conflict of interest.

\section{Bibliography}

1. Scott B Snapper. "Very-Early-Onset Inflammatory Bowel Disease". Gastroenterology and Hepatology 11.8 (2015): 554-556.

2. Holm H Uhlig. "The Diagnostic Approach to Monogenic Very Early Onset Inflammatory Bowel Disease". Gastroenterology 147.5 (2014): 990-1007.

3. Sullivan Kathleen., et al. "Very early-onset inflammatory bowel disease an integrated approach". Current Opinion in Allergy and Clinical Immunology 18.6 (2018): 459-469.
4. Jung Ok Shim. "Recent Advance in Very Early Onset Inflammatory Bowel Disease". Pediatric Gastroenterology Hepatology and Nutrition 22.1 (2019): 41-49.

5. Mohammad I El Mouzan., et al. "Incidence of Pediatric Inflammatory Bowel Disease in Saudi Arabia: A Multicenter National Study". Inflammatory Bowel Disease 20 (2014): 10851090.

6. Abdulrahman Al-Hussaini., et al. "Clinical Pattern of EarlyOnset Inflammatory Bowel Disease in Saudi Arabia: A Multicenter National Study". Inflammatory Bowel Disease 22 (2016): 1961-1970.

7. Thomas Müller., et al. "Congenital secretory diarrhoea caused by activating germline mutations in GUCY2C". Gut 65 (2016): 1306-1313.

8. Rune R Tronstad., et al. "Genetic and transcriptional analysis of inflammatory bowel disease-associated pathways in patients with GUCY2C-linked familial diarrhea". Scandinavian Journal of Gastroenterology 53 (2018): 1264-1273.

9. Fiskerstrand T., et al. "Familial diarrhea syndrome caused by an activating GUCY2C mutation". The New England Journal of Medicine 366.17 (2012): 1586-1595.

10. Johnson Nameirakpam., et al. "Genetics on early onset inflammatory bowel disease: An update". Genes and Diseases 7 (2020): 93-106.

11. Lucia De Martino., et al. "Novel Findings into AIRE Genetics and Functioning: Clinical Implications". Frontiers in Pediatrics (2016).

12. Iivo Hetema "ki., et al. "Anticommensal Responses Are Associated with Regulatory T Cell Defect in Autoimmune Polyendocrinopathy-Candidiasis-Ectodermal Dystrophy Patients". The Journal of Immunology (2016).

13. Judith R Kelsen., et al. "North American Society for Pediatric Gastroenterology, Hepatology, and Nutrition Position Paper on the Evaluation and Management for Patients with Very Earlyonset Inflammatory Bowel Disease". Journal of Pediatric Gastroenterology and Nutrition 70 (2020): 389-403.

14. Harris M., et al. "Reversible Metaphyseal Dysplasia, a Novel Bone Phenotype, in Two Unrelated Children with Autoimmune polyendocrinopathy-Candidiasis Ectodermal Dystrophy: Clinical and Molecular Studies". The Journal of Clinical Endocrinology and Metabolism 88.10 (2003): 4576-4585.

15. Maria J Gutierrez., et al. "Childhood Polyarthritis As Early Manifestation of Autoimmune Polyendocrinopathy with Candidiasis and Ectodermal Dystrophy Syndrome". Frontiers in Immunology 8 (2017): 377. 
Assets from publication with us

- Prompt Acknowledgement after receiving the article

- Thorough Double blinded peer review

- Rapid Publication

- Issue of Publication Certificate

- High visibility of your Published work

Website: www.actascientific.com

Submit Article: www.actascientific.com/submission.php

Email us: editor@actascientific.com

Contact us: +919182824667 